\title{
LOCALLY BOUNDED SETS OF HOLOMORPHIC MAPPINGS
}

\author{
JOSÉ BONET, PABLO GALINDO, DOMINGO GARCÍA AND MANUEL MAESTRE
}

\begin{abstract}
Several results and examples about locally bounded sets of holomorphic mappings defined on certain classes of locally convex spaces (Baire spaces, $(D F)$-spaces, $C(X)$-spaces) are presented. Their relation with the classification of locally convex spaces according to holomorphic analogues of barrelled and bornological properties of the linear theory is considered.
\end{abstract}

Introduction. The main topic discussed in this article is the classification of locally convex spaces according to the holomorphic analogues of barrelled, bornological and quasibarrelled properties introduced for the linear theory. This question is related to the study of locally bounded sets of holomorphic mappings defined on open subsets of locally convex spaces.

A satisfactory classification can only be obtained in the case of special classes of locally convex spaces, e.g., metrizable spaces, Baire spaces, $(D F)$-spaces and spaces of type $C(X)$. Some positive results are presented and several examples will show the essential differences between the linear and the holomorphic theory.

We shall use standard notations of locally convex spaces as in [21 and 22] and of infinite holomorphy as in [15 and 33]. The word "space" will mean separated locally convex topological vector space. If $E$ is a space, $\operatorname{cs}(E)$ denotes the set of all continuous seminorms on $E$. A family $\mathcal{X}$ of mappings defined on an open subset $U$ of a space $E$ with values in a normed space $F$ is called locally bounded if for every $x \in U$ there is a neighbourhood $V$ of $x$ contained in $U$ and $M>0$ with $\|f(y)\| \leq M$ for every $f \in \mathcal{X}$ and $y \in V$.

Now we describe briefly the organization of this paper. The first section discusses various extensions of the classical Hartogs' theorem on separate analyticity. According to results of Arias de Reyna [3] and Valdivia [39] a product of two metrizable Baire spaces need not be Baire. Consequently Theorem 1.2 is a proper extension of [6, Corollary 6.2]. In $\S 2$ we give a complete characterization of the spaces of type $C(X)$ which are holomorphically barrelled and holomorphically quasibarrelled (cf. [5]). Dineen has recently proved in [16] that every weakly holomorphic mapping defined on any open subset of $\mathbf{C}^{N} \times \mathbf{C}^{(N)}$ is holomorphic. This result provides the first example of a holomorphically Mackey space (cf. [5]) which is not holomorphically quasibarrelled. In $\S 3$ the result of Dineen is extended and more examples and properties of holomorphically Mackey spaces are given. In the last section we present various results, on spaces related to $(D F)$-spaces, concerning the following topics: sequential completeness of $H(U, F)$ endowed with the compact open topology, local boundedness of sequences of holomorphic mappings and the equivalence of local boundedness of sets and sequences on separable spaces.

Received by the editors April 14, 1986 and, in revised form, July 15, 1987.

1980 Mathematics Subject Classification (1985 Revision). Primary 46G20; Secondary 46E10. 
ACKNOWLEDGEMENTS. This article is partially supported by the CAYCIT.

We are indebted to Professor Nachbin, who stimulated our interest in infinite holomorphy during a visit to Valencia in June 1985. We want to thank Professor Dineen for the interesting discussions about this article we had in Dublin in 1985 and 1986. Galindo and Maestre gratefully acknowledge the support of the "Conselleria de Cultura, Educació i Ciencia de la Generalitat Valenciana".

Finally we would like to express our gratitude to the referee for his valuable suggestions.

1. Hartogs' theorem on Baire spaces. Let $E, F$ and $G$ be spaces, $U$ an open subset of $E \times G$ and $f: U \rightarrow F$. For every $x \in E$ we put $U_{x}:=\{y \in G:(x, y) \in U\}$ and we define $f_{x}: U_{x} \rightarrow F$ by $f_{x}(y)=f(x, y)$ for all $y \in U_{x}$. We can define analogousiy $f_{y}: U_{y} \rightarrow F$ for all $y \in G$. The mapping $f$ is said to be separately holomorphic if $f_{x}$ and $f_{y}$ are holomorphic for every $(x, y) \in U$. The classical Hartogs' theorem asserts that every separately holomorphic function defined on an open subset of a product of two Banach spaces is holomorphic (see [8, Theorem 14.27]).

1.1. THEOREM. Let $E$ be a Baire space, $G$ a metrizable space, $F$ a space and $U$ an open subset of $E \times G$. Let $f: U \rightarrow F$ be a separately holomorphic mapping which is bounded on the subsets of $U$ of the form $K \times L$, for every finite-dimensional compact subset $K$ of $E$ and every compact subset $L$ of $G$. Then $f$ is holomorphic.

ProOF. Without loss of generality we may suppose that $U=U_{1} \times U_{2}, F$ normed. Since $\mathbf{C} \times G$ is metrizable, for every $\xi \in U_{1}$ and every $x \in E$, the mapping $g_{\xi, x}(\lambda, y)=f(\xi+\lambda x, y)$ defined on $\tilde{U}:=\left\{\lambda \in \mathbf{C}: \xi+\lambda x \in U_{1}\right\} \times U_{2}$ is holomorphic (cf. [5, Proposition 6]). Fix $(a, b) \in U$. Let $W_{1} \times W_{2}$ be an open absolutely convex subset of $E \times G$ such that $(a, b)+W_{1} \times W_{2} \subset U$. If $\left(V_{n}\right)$ is a decreasing basis of absolutely convex neighbourhoods of the origin in $G$ with $V_{1} \subset W_{2}$, we set $B_{n}:=\left\{x \in W_{1}:\left\|(1 / m !) \hat{d}^{m} f(a, b)(x, y)\right\| \leq n\right.$ for every $y \in V_{n}$ and $\left.m=0,1,2, \ldots\right\}$.

Given $y \in W_{2}$, we define $\tilde{f}: W_{1} \times\left\{\lambda y: \lambda \in \mathbf{C}\right.$ and $\left.\lambda y \in W_{2}\right\} \rightarrow F$ by $\tilde{f}(x, \lambda y):=$ $f((a, b)+(x, \lambda y))$. According to [38, I§1.2(10)] $E \times\{\lambda y: \lambda \in \mathbf{C}\}$ is a Baire space, therefore we can apply [24, Theorem 2.3] to obtain that $\tilde{f}$ is holomorphic. Hence $(1 / m !) \hat{d}^{m} \tilde{f}(0,0)$ is continuous for every $m=0,1, \ldots$ Moreover

$$
(1 / m !) \hat{d}^{m} \tilde{f}(0,0)(x, y)=(1 / m !) \hat{d}^{m} f(a, b)(x, y)
$$

for every $x \in W_{1}$ and consequently the mapping $(1 / m !) \hat{d}^{m} f(a, b)(\cdot, y)$ is continuous on $W_{1}$, hence $B_{n}$ is closed in $W_{1}$.

Now we show that $W_{1}$ is the union of $\left(B_{n}: n=1,2, \ldots\right)$. Assume the existence of $x \in W_{1}$ such that for each $m \in \mathbf{N}$ there is $\lambda_{m} \in \mathbf{C}$ with $\left|\lambda_{m}\right| \leq 1$ and $y_{m} \in V_{m}$ such that $\left\|f\left((a, b)+\left(\lambda_{m} x, y_{m}\right)\right)\right\| \geq m$. Then $g_{a, x}$ is unbounded on the compact set $\{\lambda x:|\lambda| \leq 1\} \times\left\{\left(b+y_{m}: m=1,2, \ldots\right) \cup(b)\right\}$ and this is a contradiction. Consequently for each $x \in W_{1}$ there is $n \in \mathbf{N}$ such that $\|f((a, b)+(\lambda x, y))\| \leq n$ for every $\lambda \in \mathbf{C}$ with $|\lambda| \leq 1$ and $y \in V_{n}$. By [15, Lemma 1.13], we have

$$
\begin{aligned}
\left\|(1 / m !) \hat{d}^{m} f(a, b)(x, y)\right\| & \leq \sup \left\{\left\|(1 / m !) \hat{d}^{m} f(a, b)(\lambda x, z)\right\|:|\lambda| \leq 1, z \in V_{n}\right\} \\
& \leq \sup \left\{\|f((a, b)+(\lambda x, z))\|:|\lambda| \leq 1, z \in V_{n}\right\} \leq n
\end{aligned}
$$

for every $m=0,1, \ldots$, hence $x \in B_{n}$. 
According to Baire's theorem we can find $s \in \mathbf{N}$ such that $B_{s}$ has nonempty interior. Hence there are $x_{0} \in B_{s}$ and an absolutely convex neighbourhood of the origin $V$ in $E$ with $x_{0}+V \subset B_{s}$. By the maximum principle $\left\|(1 / m !) \hat{d}^{m} f(a, b)(x, y)\right\| \leq s$ for every $(x, y)$ in $V \times V_{s}$ and every $m=0,1, \ldots$ Now Taylor series expansion at $(a, b)$ shows that $f$ is bounded on $(a, b)+\frac{1}{2}\left(V \times V_{s}\right)$.

1.2. THEOREM. Let $E$ be a Baire space, $G$ a metrizable Baire space and $U$ an open subset of $E \times G$. If $f: U \rightarrow F$ is separately holomorphic, then $f$ is holomorphic.

Proof. Let $K$ be a finite-dimensional compact subset of $E$ and $L$ a compact subset of $G$ such that $K \times L$ is contained in $U$. We put $\tilde{E}$ for the linear span of $K . \tilde{E} \times G$ is a Baire space by $[\mathbf{3 8}, \mathrm{I} \S 1.2(10)]$, hence, if $\tilde{f}$ is the restriction of $f$ to $\tilde{U}:=U \cap(\tilde{E} \times G)$, it follows from [24, Theorem 2.3] that $\tilde{f}$ is holomorphic. Since $K \times L$ is a compact subset of $\tilde{U}, f$ is bounded on $K \times L$. The conclusion follows from Theorem 1.1.

Now we turn to the holomorphic classification of spaces. Following Barroso, Matos and Nachbin [5] a space $E$ is said to be holomorphically (quasi)barrelled if for every open subset $U$ of $E$, every family of $H(U, F)$ which is bounded on the finite-dimensional compact (compact) subsets of $U$ is locally bounded. Every Baire space is holomorphically barrelled (see [5, Proposition 37]) and every metrizable space is holomorphically quasibarrelled [5, Proposition 54].

1.3. Proposition. Let $E$ be a Baire space, $G$ a metrizable holomorphically barrelled space and $U$ an open subset of $E \times G$. If $f: U \rightarrow F$ is separately holomorphic, then $f$ is holomorphic.

Proof. We may suppose that $U=U_{1} \times U_{2}$. Let $K, L$ and $f_{x}: U_{2} \rightarrow F, x \in K$, denote the same as in Theorem 1.1. Obviously the family $\left\{f_{x}: x \in K\right\}$ is contained in $H\left(U_{2}, F\right)$ and it is bounded on the finite-dimensional compact subsets of $U_{2}$, hence it is locally bounded. Thus $f$ is bounded on $K \times L$ and the conclusion follows from Theorem 1.1.

1.4. Proposition. If $E_{1}, E_{2}, \ldots, E_{n}$ are metrizable Baire spaces, then $E_{1} \times$ $E_{2} \times \cdots \times E_{n}$ is holomorphically barrelled.

ProOF. The proof follows by induction, using Proposition 1.3, once the case $n=2$ is established. Let $U$ be an open subset of $E_{1} \times E_{2}$ and $\mathcal{X}$ a subset of $H(U, F)$ which is bounded on the finite-dimensional compact subsets of $U$. We define $f: U \rightarrow$ $l_{\infty}(X, F)$ by $f(x):=(g(x): g \in \mathcal{X})$. Since $E_{1}$ and $E_{2}$ are holomorphically barrelled, $f$ is separately holomorphic, and the conclusion follows form Proposition 1.3.

1.5. Proposition. If $E$ is a Baire space and $G$ is metrizable, then $E \times G$ is holomorphically quasibarrelled.

ProOF. Let $U$ be an open subset of $E \times G$ and $X$ a subset of $H(U, F)$ which is bounded on the compact subsets of $U$. We define $f$ from $U$ into $l_{\infty}(\mathcal{X}, F)$ by $f(x):=(g(x): g \in \mathcal{X})$. Since $E$ and $G$ are holomorphically quasibarrelled, $f$ is separately holomorphic. Applying Theorem 1.1 the conclusion follows.

1.6. EXAMPLES. Holomorphically quasibarrelled spaces which are neither bornological nor barrelled. 
(a) Let $E$ be a nonbornological Baire space and $G$ a nonbarrelled metrizable space. According to Proposition 1.5, $E \times G$ is holomorphically quasibarrelled but it is neither barrelled nor bornological.

(b) Let $\left(E_{i}: i \in I\right)$ be a family of metrizable spaces, at least one of them not being barrelled. Assume that the cardinal of $I$ is the continuum. Let $H$ be the subspace of $E:=\pi\left(E_{i}: i \in I\right)$ of all the elements with countably many coordinates distinct from zero. Take $x \in E \backslash H$ and set $G:=H \oplus \operatorname{span}(x)$. Using a result of Noble $[\mathbf{1 7}$, p. 270] one can show that $G$ is holomorphically quasibarrelled (see $[\mathbf{1 9}$, Remark 17], and [33, Chapter 12]). It is proved in [37] that $E$ is neither barrelled nor bornological.

Theorem 1.1 generalises [25, Theorem 3.1], Theorem 1.2 generalises [5, Corollary 6.2], and Proposition 1.4 has been recently extended to infinite products in [23].

2. Sets of holomorphic mappings on spaces of continuous functions. In this section $X$ stands for a completely regular Hausdorff topological space and $C(X)$ for the linear space of all continuous functions defined on $X$ with values in $\mathbf{C}$. The compact open topology on $C(X)$ will be denoted by $t_{0}$. A subset $B$ of $X$ is called $C$-bounding (resp. Warner-bounded) if for every $u \in C(X)$ (resp. bounded subset $A$ of $\left.\left(C(X), t_{0}\right)\right), \sup (|u(x)|: x \in B)($ resp. $\sup (|u(x)|: x \in B$ and $u \in A))$ is finite.

The theorem of Nachbin and Shirota $[\mathbf{2 8}$ and $\mathbf{3 5}]$ characterizes the barrelledness of $\left(C(X), t_{0}\right)$ in the following sense. $\left(C(X), t_{0}\right)$ is barrelled if and only if every $C$ bounding subset of $X$ is relatively compact. The topological spaces satisfying this property are called $\mu$-spaces or NS-spaces. Warner proved in [40] that $\left(C(X), t_{0}\right)$ is quasibarrelled if and only if every Warner-bounded subset of $X$ is relatively compact.

2.1. THEOREM. Let $X$ be a $\mu$-space and $U$ an open subset of $\left(C(X), t_{0}\right)$. If $\mathcal{X}$ is a subset of $H(U)$ which is bounded on the finite-dimensional compact subsets of $U$, then $\mathcal{X}$ is locally bounded.

ProOF. Given $x_{0} \in U$, there are a compact subset $K$ of $X$ and a positive real number $\varepsilon$ such that the set $B(K, \varepsilon):=\{u \in C(X):|u(x)|<\varepsilon$ for each $x \in K\}$ is contained in $U-x_{0}$, i.e. $x_{0}+B(K, \varepsilon) \subset U$. Now the Cauchy inequalities ensure that the family $\tilde{X}:=\left\{(1 / m !) \hat{d}^{m} f\left(x_{0}\right): f \in \mathcal{X}, m=0,1, \ldots\right\}$ is bounded on the finitedimensional compact subsets of $B(K, \varepsilon)$, hence on the fast convergent sequences contained in $B(K, \varepsilon)$ by [29, Lemma 11].

For each $P \in \tilde{X}$ we set $W_{P}:=\left\{x \in X\right.$ : for every neighbourhood $V_{x}$ of $x$, there are $u, v \in C(X)$ with supp $v \subset V_{x}$ such that $\left.P(u+v) \neq P(v)\right\}$. We shall see that $W:=\overline{\bigcup\left(W_{P}: P \in \mathcal{X}\right)}$ is compact. Suppose that this is not the case. Since $X$ is a $\mu$-space, there is $u \in C(X)$ such that $A_{n}:=\{x \in X:|u(x)|>n\}$ meets $W$ for every $n \in \mathbf{N}$. Therefore, for each $n \in \mathbf{N}$, there is $P_{n} \in \tilde{X}$ such that $W_{P_{n}} \cap A_{n} \neq \varnothing$ and consequently we can find $x_{n} \in W_{P_{n}}$ with $\left|u\left(x_{n}\right)\right|>n$. As $x_{n} \in W_{P_{n}}$, there are $u_{n}, v_{n}$ in $C(X)$ with $P_{n}\left(u_{n}+v_{n}\right) \neq P_{n}\left(v_{n}\right)$ and supp $v_{n} \subset A_{n}$. Moreover, we can suppose $u_{n}$ bounded on $X$ (since the bounded continuous functions on $X$ form a dense subset of $\left(C(X), t_{0}\right)$ ) and even $\left|u_{n}(x)\right| \leq\left(n 2^{n}\right)^{-1}$ for each $x \in X$ (to obtain the former inequality it is enough to multiply both functions $u_{n}$ and $v_{n}$ by an adequate scalar). Since $z \rightarrow P_{n}\left(u_{n}+z v_{n}\right)$ defines an entire function on $\mathbf{C}$ which is not constant, there is $\beta_{n} \in \mathbf{C}$ such that $\left|P_{n}\left(u_{n}+\beta_{n} v_{n}\right)\right|>n$. 
The series $\sum_{n=1}^{\infty} n\left|u_{n}\right|$ converges uniformly, hence it defines a continuous function $w_{1}$ and the function $w_{2}$ defined by the series $\sum_{n=1}^{\infty} n\left|\beta_{n} v_{n}\right|$ is also continuous since $\left\{A_{n}: n \in \mathbf{N}\right\}$ is locally finite. Then $w_{0}:=w_{1}+w_{2}$ is a continuous nonnegative function on $X$. Therefore the set $D:=\left\{w \in C(X):|w| \leq w_{0}\right\}$ is a Banach disc in $C(X)$ (cf. [10]). Furthermore

$$
\left|u_{n}+\beta_{n} v_{n}\right|=(1 / n)\left|n u_{n}+n \beta_{n} v_{n}\right| \leq(1 / n) w_{0},
$$

hence the sequence $\left(u_{n}+\beta_{n} v_{n}: n \in \mathbf{N}\right)$ converges to the origin in the Banach space $C(X)_{D}$ and, of course, in $\left(C(X), t_{0}\right)$. Therefore there is $p \in \mathbf{N}$ such that $\left(u_{n}+\beta_{n} v_{n}: n=p, p+1, \ldots\right)$ is a fast convergent sequence contained in $B(K, \varepsilon)$ and consequently $\tilde{X}$ must be bounded on it, but this is a contradiction.

Now, proceeding as in the proof of Proposition 6.29 of [15], we obtain that every $P \in \tilde{X}$ factors through $C(W)$, i.e. there is $\bar{P} \in P(C(W))$ such that $P(\varphi)=\bar{P}(\varphi \mid W)$ for every $\varphi \in C(X)$. The family $\{\bar{P}: P \in \tilde{X}\}$ is bounded on the finite-dimensional compact subsets of the open subset $\{\varphi \mid W: \varphi \in B(K, \varepsilon)\}$ of the Banach space $C(W)$, hence $\{\bar{P}: P \in \tilde{X}\}$ is locally bounded by [5, Proposition 37]. Therefore $\tilde{X}$ is bounded on a certain neighbourhood $V$ of the origin in $\left(C(X), t_{0}\right)$. Using Taylor series expansion of the elements of $\mathcal{X}$ we obtain that $X$ is bounded on $x_{0}+\frac{1}{2} V$.

According to the definition of holomorphically barrelled space and [5, Proposition 35], we have

2.2. COROLlaRY. $X$ is a $\mu$-space if and only if $\left(C(X), t_{0}\right)$ is holomorphically barrelled.

2.3. REMARK. The idea of the proof of Theorem 2.1 (i.e. the construction and use of the sets $W_{P}$ ) first occurs in [13, Proposition 7.11] and is also used in [36]. The following theorem is Example 8.6 of [13].

2.4. THEOREM. $\left(C(X), t_{0}\right)$ is holomorphically quasibarrelled if and only if every Warner-bounded subset of $X$ is relatively compact.

A space $E$ is called holomorphically (ultra)bornological if, given any space $F$ and any open subset $U$ of $E$, a $G$-holomorphic mapping $f: U \rightarrow F$ bounded on the (fast) compact subsets of $U$ is holomorphic $[\mathbf{5}, \mathbf{1 9}]$. The relationship between the holomorphic spaces defined in this paper is the same as the relationship between the corresponding linear spaces. We refer to $[5,19$ and 33$]$ for details.

2.5. Proposition. Let $E$ be a (DFM)-space. The following conditions are equivalent.

(i) $H_{M}(U, F)=H(U, F)$ for each open subset $U$ of $E$ and each space $F$.

(ii) $E$ is holomorphically ultrabornological.

(iii) $E$ is holomorphically barrelled.

ProOF Let $\left(B_{n}: n \in \mathrm{N}\right)$ be a fundamental increasing sequence of compact subsets of $E$. A $G$-holomorphic mapping $f: U \rightarrow F$ belongs to $H_{M}(U, F)$ if and only if it maps Mackey convergent sequences onto convergent sequences or equivalently if it is continuous on the open subset $U \cap E_{B_{n}}$ of the Banach space $E_{B_{n}}$ for each $n \in \mathbf{N}$ or if and only if it maps convergent sequences onto bounded sequences, since each $E_{B_{n}}$ is holomorphically ultrabornological. Therefore (i) and (ii) are equivalent and it only remains to show that (iii) implies (ii). If $f: U \rightarrow F$ is a $G$-holomorphic 
mapping which is bounded on the fast convergent sequences of $U$ and $x_{0} \in U$, we have that $\hat{d}^{m} f\left(x_{0}\right)$ is a continuous polynomial on $E$, since $\hat{d}^{m} f\left(x_{0}\right)(z)=\hat{d}^{m}(f \mid U \cap$ $\left.E_{B_{n}}\right)\left(x_{0}\right)(z)$ for every $z \in E_{B_{n}}$ and large enough $n \in \mathbf{N}$, and since the inductive limit of the spaces $E_{B_{n}}$ in the category of locally convex spaces and polynomial mappings coincides with $E$ (cf. [14, Corollary 11]). Moreover the family $\chi:=$ $\left\{(1 / m !) \hat{d}^{m} f\left(x_{0}\right): m=0,1,2, \ldots\right\}$ is bounded on the finite-dimensional compact subsets of a certain neighbourhood of the origin. Therefore there is a neighbourhood $V$ of the origin in $E$ on which $X$ is bounded, since $E$ is holomorphically barrelled. Now the Taylor series expansion of $f$ at $x_{0}$ converges uniformly on $x_{0}+\frac{1}{2} V$, hence $f$ is continuous at $x_{0}$.

3. When is a weakly holomorphic mapping also holomorphic? Holomorphically Mackey spaces. Let $U$ be an open subset of a space $E$ and $F$ a space. We let $F_{\sigma}$ denote the space $F$ endowed with the weak topology $\sigma\left(F, F^{\prime}\right)$. A mapping $f: U \rightarrow F$ is called weakly holomorphic if $f: U \rightarrow F_{\sigma}$ is holomorphic. In several situations it is important to know if a weakly holomorphic mapping is already holomorphic (see [15, Chapter 2, 14 and 5]). This section is strongly inflenced by the recent work of Dineen [16]. We shall extend some of his results with a slightly different approach.

The notation and terminology of open surjective limits will be used as in [13 and 16, Chapter 6].

3.1. PROPOSITION. Let $\left(E_{n}: n \in \mathbf{N}\right)$ be a sequence of spaces in which the weakly holomorphic mappings defined on open sets are holomorphic. If $E$ is the directed open surjective limit of this sequence under the sequence of mappings $\left(\pi_{n}: E\right.$ $\left.\rightarrow E_{n} ; n \in \mathbf{N}\right)$, then $H(U, F)=H\left(U, F_{\sigma}\right)$ for every open subset $U$ of $E$ and every space $F$.

ProOF. Let $f: U \rightarrow F$ be a weakly holomorphic mapping. We may suppose, without loss of generality, that $U$ is an absolutely convex open subset of $E, F$ is a Banach space, $U=\pi_{1}^{-1}\left(\pi_{1}(U)\right)$ and ker $\pi_{n+1}$ contained in ker $\pi_{n}$ (because $E$ is the directed open surjective limit). According to our assumptions it suffices to show that $f$ factors through some $E_{n}$. If this is not the case, for each $n \in \mathbf{N}$ there are $x_{n} \in U$ and $y_{n} \in E$ such that $\pi_{n}\left(y_{n}\right)=0$ and $f\left(x_{n}+y_{n}\right) \neq f\left(x_{n}\right)$. For each $n \in \mathbf{N}$, the mapping $\lambda \in \mathbf{C} \rightarrow f\left(x_{n}+\lambda y_{n}\right)-f\left(x_{n}\right)$ is a nonconstant entire mapping, hence, by Liouville's theorem, we can choose $\lambda_{n} \in \mathbf{C}$ such that $\left\|f\left(x_{n}+\lambda_{n} y_{n}\right)-f\left(x_{n}\right)\right\|>n$. On the other hand, given $\varphi \in F^{\prime}$, the mapping $\varphi \circ f$ is holomorphic, hence it factors through some $E_{m}$ and therefore $\varphi \circ f\left(x_{n}+\lambda_{n} y_{n}\right)-\varphi \circ f\left(x_{n}\right)=0$ for all $n \geq m$. Thus the sequence $\left(f\left(x_{n}+\lambda_{n} y_{n}\right)-f\left(x_{n}\right): n \in \mathbf{N}\right)$ is weakly convergent to the origin. This is a contradiction.

3.2. COROLLARY. Let $E$ be the strict inductive limit of a sequence of FréchetMontel spaces. Then $H(U, F)=H\left(U, F_{\sigma}\right)$ for every open subset of the strong dual of $E$ and every space $F$.

According to [5] a space $E$ is called holomorphically Mackey if for every open subset $U$ of $E$ and every space $F$, each weakly holomorphic mapping $f: U \rightarrow F$ is holomorphic. Clearly every holomorphically Mackey space is endowed with its Mackey topology. By [5, Proposition 74], every holomorphically quasibarrelled space is holomorphically Mackey. The converse of this result is false as was recently 
established by Dineen, [16], by proving that $\mathbf{C}^{N} \times \mathbf{C}^{(N)}$ is holomorphically Mackey. Proposition 3.1 and Corollary 3.2 above are extensions of this result of Dineen.

3.3. PROPOSITION. If $E$ is holomorphically quasibarrelled, then $E \times \mathbf{C}^{N}$ is holomorphically Mackey.

PROOF. It is easily seen that $E \times \mathbf{C}^{n}$ is holomorphically quasibarrelled for any positive integer $n$ and hence it is holomorphically Mackey. An application of Proposition 3.1 completes the proof.

3.4. EXAMPLE. For every $(D F M)$-space $E, E \times \mathrm{C}^{N}$ is holomorphically Mackey. Observe that $E \times \mathbf{C}^{N}$ is not holomorphically quasibarrelled if $E$ does not have a total bounded subset. Indeed, if $E$ does not have a total bounded subset, then $\mathbf{C}^{(N)} \times \mathbf{C}^{N}$ is a complemented subspace of $E \times \mathbf{C}^{N}$, now apply [5, Example 18] and $[19$, Remark 6$]$.

3.5. Proposition. If $\left(E_{i}: i \in I\right)$ is a family of spaces such that all finite products of $E_{i}$ 's are holomorphically Mackey, then $E:=\pi\left(E_{i}: i \in I\right)$ is holomorphically Mackey.

ProOF. It suffices to show that each $f \in H\left(U, F_{\sigma}\right)$ where $U$ is an absolutely convex neighbourhood of the origin in $E$ and $F$ is a Banach space, factors through $E_{J}:=E_{i_{1}} \times E_{i_{2}} \times \cdots \times E_{i_{s}}$ where $i_{n} \in I$ for all $n$. If this were not true then we could find a countable subset $J^{\prime}$ of $I$ such that $f$ does not factor through $\pi\left(E_{i}: i \in J^{\prime \prime}\right)$ for any finite subset $J^{\prime \prime}$ of $J^{\prime}$. If we let $F=\pi\left(E_{i}: i \in J^{\prime}\right)$ then $F$ is an open directed (by the finite subsets of $J^{\prime}$ ) surjective limit of holomorphically Mackey spaces and hence, by Proposition 3.1, it is also holomorphically Mackey. This contradicts our choice of $J^{\prime}$ and completes the proof.

3.6. COROLlARY. If $E$ is holomorphically quasibarrelled, then $E \times \mathbf{C}^{I}$ is holomorphically Mackey for every infinite index set I.

The following example is based on [5, Lemma 19] and [16, Proposition 2].

3.7. EXAMPLE. If $I$ is an index set with cardinal larger than or equal to the continuum, then there is a noncontinuous 2-homogeneous polynomial $P: C^{(I)} \rightarrow$ $l_{2}(I \times I)$ such that $P: C^{(I)} \rightarrow\left(l_{2}(I \times I), \sigma\left(l_{2}(I \times I), l_{2}(I \times I)\right)\right)$ is continuous. As in [5, Lemma 19], consider a set $S$ of functions $s: I \rightarrow \mathbf{N}$ such that for every function $t: I \rightarrow \mathbf{R}_{+}$there is some $s \in S$ for which $s \leq c \cdot t$ is false for all $c \in \mathbf{R}_{+}$ and $S$ having cardinal equal to the continuum. According to the cardinality of $I$, there is a surjective mapping $i \in I \rightarrow s_{i} \in S$. Define $r(i, j):=\max \left(s_{i}(j), s_{j}(i)\right)$ for $(i, j) \in I \times I$ and the symmetric bilinear mapping $f: \mathbf{C}^{(I)} \times \mathbf{C}^{(I)} \rightarrow l_{2}(I \times I)$ by

$$
f(x, y)=\sum\left(r(i, j) x_{i} y_{j} e_{i j}:(i, j) \in I \times I\right)
$$

for $x=\left(x_{i}: i \in I\right), y=\left(y_{i}: i \in I\right) \in \mathbf{C}^{(I)}$, where $e_{i j}$ is the $(i, j)$ th unit vector in $l_{2}(I \times I)$. Let $P: \mathbf{C}^{(I)} \rightarrow l_{2}(I \times I)$ be the 2-homogeneous polynomial given by $P(x)=f(x, x)$ for $x \in \mathbf{C}^{(I)}$. We claim that $P$ is weakly continuous. If $\gamma=$ $\left(\gamma_{i j}:(i, j) \in I \times I\right) \in l_{2}(I \times I)^{\prime}$, the set $A=\left\{i \in I:\right.$ there is $j \in I$ with $\gamma_{i j} \neq 0$ or $\left.\gamma_{j i} \neq 0\right\}$ is countable. Since $\mathbf{C}^{(A)}=\mathbf{C}^{(N)}$, it follows from [15, Ex. 1.63], that $(\gamma \circ P) \mid \mathbf{C}^{(A)}$ is holomorphic, hence $\gamma \circ P$ is holomorphic. Proceeding as in the proof of [5, Lemma 19] one can prove that $P$ is not continuous.

In particular this example shows that $\mathbf{C}^{(I)}$ is not holomorphically Mackey. 
4. Countable sets of holomorphic mappings. When is $H(U)$ sequentially complete for the compact open topology?. Grothendieck introduced in [20] the $(D F)$-spaces as a generalisation of strong duals of Fréchet spaces. Many authors have studied conditions on a space $E$ to ensure that a countable Banach Steinhaus theorem holds for $E$. More precisely, to ensure that a sequence of linear mappings defined on $E$ which is bounded for some topology of the uniform convergence is equicontinuous. An exhaustive and deep study of these conditions is presented in [21, Chapter 12].

In $[\mathbf{2 1}, 12.2 .1]$ it is proved that the following conditions are equivalent for a space $E$. (i) Every (bornivorous) barrel in $E$ which is the intersection of a sequence of absolutely convex neighbourhoods of the origin is a neighbourhood of the origin. (ii) For every space $F$, any sequence of linear continuous mappings which is (strongly) simply bounded is equicontinuous. Therefore, every $(D F)$-space satisfies a countable Banach Steinhaus theorem.

In the holomorphic context, according to an example in [5, 18], and [11, 3.4], we know that if $E$ is an infinite-dimensional Banach space, then $H\left(E \times \mathbf{C}^{(N)}\right)$ is not sequentially complete for the compact open topology, consequently there is a sequence $\left(f_{n}: n \in \mathbf{N}\right)$ in $H\left(E \times \mathbf{C}^{(N)}\right)$ which is bounded on the compact sets but not locally bounded. On the other hand, $E \times \mathbf{C}^{(N)}$ is a $(D F)$-space. Therefore a "countable Banach Steinhaus theorem for holomorphic mappings" (i.e. "every countable subset of $H(U, F)$ which is bounded for the compact open topology is locally bounded") does not hold in general for $(D F)$-spaces.

In this section we present various results, on spaces rather similar to $(D F)$ spaces, concerning the following topics; sequential completeness of $H(U, F)$ for the compact open topology, which will be denoted by $\tau_{0}$, local boundedness of sequences of holomorphic mappings and the equivalence of local boundedness of sets and sequences on separable spaces.

First note that since the projective tensor product of $(D F)$-spaces is again a $(D F)$-space it follows that every sequence of $m$-homogeneous polynomials on a $(D F)$-space which is $\tau_{0}$-bounded is also locally bounded (see e.g. [1]). On the other hand, it is easily seen that if every $\tau_{0}$-bounded sequence in $H(U, F)$ is locally bounded and $F$ is sequentially complete then $\left(H(U, F), \tau_{0}\right)$ is also sequentially complete. The following result is a form of converse to this statement.

4.1. Proposition. Let $E$ be a $(D F)$-space. If $\left(H(U, F), \tau_{0}\right)$ is sequentially complete for every open subset $U$ of $E$ and every Banach space $F$, then every bounded sequence in $\left(H(U, F), \tau_{0}\right)$ is locally bounded for every $U$ and $F$ as above.

ProOF. Let $\left(f_{n}: n \in \mathbf{N}\right)$ be a bounded sequence in $\left(H(U, F), \tau_{0}\right)$. We define $g: U \rightarrow l_{\infty}(F)$ by $g(u):=\left(f_{n}(u): n \in \mathbf{N}\right)$. Clearly $g$ is bounded on the compact subsets of $U, G$-holomorphic and

$$
(1 / m !) \hat{d}^{m} g(\xi)(x)=\left((1 / m !) \hat{d}^{m} f_{n}(\xi)(x): n \in \mathbf{N}\right)
$$

for every $x \in E$ and $\xi \in U$. Take $\xi \in U$ and select an absolutely convex open neighbourhood of the origin $W$ in $E$ with $\xi+W \subset U$. The Cauchy inequalities ensure that the sequence $\left((1 / m !) \hat{d}^{m} f_{n}(\xi): n \in \mathbf{N}\right)$ in $\mathcal{P}\left({ }^{m} E, F\right)$ is bounded on the compact subsets of $W$. Hence, since $E$ is a $(D F)$-space, this sequence is locally bounded. Therefore $(1 / m !) \hat{d}^{m} g(\xi)$ belongs to $P\left({ }^{m} E, l_{\infty}(F)\right)$. The Cauchy inequalities applied to $g$ show that the Taylor series expansion of $g$ converges uniformly on 
the compact subsets of $\xi+W$. By assumption, $\left(H\left(\xi+W, l_{\infty}(F)\right), \tau_{0}\right)$ is sequentially complete, hence $g \in H\left(\xi+W, l_{\infty}(F)\right)$. This implies that $\left(f_{n}: n \in \mathbf{N}\right)$ is locally bounded.

A space $F$ is said to satisfy the countable neighbourhood property (c.n.p.) if for every sequence $\left(p_{n}: n \in \mathbf{N}\right) \subset \operatorname{cs}(E)$ there are $p \in \operatorname{cs}(E)$ and $\lambda_{n}>0, n \in \mathbf{N}$, with $p_{n} \leq \lambda_{n} p$. Every $(g D F)$-space satisfies the c.n.p. (see [7, 18 and 21] for details).

4.2. Proposition. Let $(E, t)$ be an open surjective limit of Banach spaces with the c.n.p. Then every sequence in $H(U, F), U$ an open subset of $E$ and $F$ a normed linear space, which is uniformly bounded on the finite-dimensional compact subsets of $U$ is locally bounded.

PROOF. It suffices to consider the case when $U$ is absolutely convex. If $\left(f_{n}: n \in\right.$ $\mathbf{N}$ ) is a sequence which is uniformly bounded on the finite-dimensional compact subsets of $U$, then each $f_{n}$ factors through one of the spaces (as $E$ is the open surjective limit) or, equivalently, it is $p_{n}$-continuous for some $t$-continuous seminorm $p_{n}$ on $E$. If $p$ is a $t$-continuous seminorm on $E$ and $p_{n} \leq \lambda_{n} p$ for some $\lambda_{n} \in \mathbf{R}_{+}$ then each $f_{n}$ is $p$-continuous and the proof is now completed by applying the fact that the required result is true for Banach spaces.

4.3. EXAMPLES. The following are classes of spaces satisfying the assumptions of 4.2 which are not quasibarrelled.

(i) $(E, t)$ for every nonseparable reflexive Banach space $E$ endowed with the topology $t$ of the uniform convergence on the separable bounded subsets of $E^{\prime}$.

(ii) $\left(C(X), t_{0}\right)$ for every noncompact completely regular Hausdorff space $X$ such that if $\left(K_{n}: n \in \mathbf{N}\right)$ is a sequence of compact subsets of $X$, then $\bigcup\left(K_{n}: n \in \mathbf{N}\right)$ is relatively compact.

(iii) $\left(c_{0}(I), t\right)$ for an uncountable index set $I$ endowed with the topology $t$ defined by the family of seminorms $\left\{p_{J}: J\right.$ a countable subset of $\left.I\right\}$, where $p_{J}(x):=$ $\sup (|x(i)|: i \in J)$ for $x \in c_{0}(I)$.

ProOF. (i) If $p$ is a $t$-continuous seminorm on $E$ then there is a separable bounded subset $B_{1}$ of $E^{\prime}$ such that $p(x)=\sup \left(|\varphi(x)|: \varphi \in B_{1}\right)$ for all $x \in E$. Let $F$ denote the separable subspace of $\left(E^{\prime},\|\cdot\|\right)$ generated by $B_{1}$ and let $B$ denote the unit ball of $E^{\prime}$. Then $p$ is equivalent to both uniform convergence on $B \cap F$ and on $B \cap \bar{F}$, the closure taken in $\left(E^{\prime}, \sigma\left(E^{\prime}, E\right)\right)$. Since the span of a countable set of separable subspaces of $F$ is also separable this shows that $(E, t)$ has the c.n.p. This shows also that $E / \operatorname{ker} p$ with the quotient norm is isomorphic to $F^{\perp}=\{x \in E: \varphi(x)=0$ for every $\varphi \in F\}$ with the norm induced by the norm of $E$. Hence it is a Banach space and this completes the proof in this case.

(ii) Every $\left(C(X), t_{0}\right)$ is an open surjective limit of Banach spaces and the condition given implies by $[\mathbf{4 0}]$ that the space is $(D F)$ and hence has the c.n.p.

(iii) See for instance $[\mathbf{1 5}]$.

We give an example of a space $E$ such that $\left(H(U), \tau_{0}\right)$ is sequentially complete for all open subsets $U$ of $E$ and yet there is a Banach space $F_{0}$ such that $\left(H\left(E, F_{0}\right), \tau_{0}\right)$ is not sequentially complete.

4.4. EXAMPLE. Let $I$ be an index set which is the increasing union of a sequence $\left(I_{n}: n \in \mathbf{N}\right)$ of subsets such that the cardinal of $I_{n+1} \backslash I_{n}$ is the continuum. On $c_{0}(I)$ we define the following seminorms. For each $n \in \mathbf{N}, p_{n}(x):=\sup \left(|x(i)|: i \in I_{n}\right)$ and for each countable subset $J$ of $I, p_{J}(x):=\sup (|x(i)|: i \in J)$ for $x \in c_{0}(I)$. We 
denote by $s$ the locally convex topology on $c_{0}(I)$ defined by the system of seminorms $\left(p_{n}: n \in \mathbf{N}\right) \cup\left(p_{J}: J \subset I\right.$ countable $)$, and we write $E$ for $\left(c_{0}(I), s\right)$. Clearly $s$ is finer than the topology $t$ given in Example 4.3(iii) and coarser than the sup-norm topology on $c_{0}(I)$. Proceeding as in $[7,1.7], E$ does not satisfy the c.n.p., hence it is not a $(g D F)$-space (cf. [21, Chapter 12]). Therefore we can apply [21, 12.4.2], to obtain a Banach space $F_{0}$ such that $L_{b}\left(E, F_{0}\right)$ is not sequentially complete, hence there is a Cauchy sequence of continuous linear mappings $g_{n}: E \rightarrow F_{0}, n \in \mathbf{N}$, in $L_{b}\left(E, F_{0}\right)$ which does not converge. Then $\left(H\left(E, F_{0}\right), \tau_{0}\right)$ is not sequentially complete. For if it were, $\left(g_{n}: n \in \mathbf{N}\right)$ being a Cauchy sequence in $\left(H\left(E, F_{0}\right), \tau_{0}\right)$, it would converge to a certain $g \in H\left(E, F_{0}\right)$. But then $g$ would be a limit of $\left(g_{n}: n \in \mathbf{N}\right)$ in $L_{b}\left(E, F_{0}\right)$.

Now we prove that every sequence $\left(f_{n}: n \in \mathbf{N}\right)$ in $H(U)$ bounded on the finitedimensional compact subsets of an open subset $U$ of $E$ is locally bounded. This clearly implies that $\left(H(U), \tau_{0}\right)$ is sequentially complete. We fix $\xi \in U$. Then $\left((1 / m !) \hat{d}^{m} f_{n}(\xi): n \in N, m=0,1,2, \ldots\right)$ is a countable family of continuous polynomials on $E$, hence on $c_{0}(I)$ for the sup-norm topology and, by [5, Lemma 25], on $\left(c_{0}(I), t\right)$. According to $[\mathbf{9}$, Lemma 3.4], we can find a countable subset $J$ of $I$ such that if $x, x^{\prime} \in c_{0}(I)$ and $p_{J}\left(x-x^{\prime}\right)=0$, then $\hat{d}^{m} f_{n}(\xi)(x)=\hat{d}^{m} f_{n}(\xi)\left(x^{\prime}\right)$ for each $m$ and $n$. Since $U$ is an open subset of $E$ there are a countable subset $K$ of $I, r \in \mathbf{N}$ and $\varepsilon>0$ with $\xi+\left\{x \in c_{0}(I): p_{K}(x)+p_{r}(x)<\varepsilon\right\} \subset U$. We set $L:=J \cup K \cup I_{r}$ and consider the continuous seminorm

$$
p_{L}(x):=\sup (|x(i)|: i \in L) .
$$

Setting $W:=\left\{x \in c_{0}(I): p_{L}(x)<\varepsilon / 2\right\}$, we have that $\xi+W \subset U$ and if $p_{L}\left(x-x^{\prime}\right)=$ 0 , then $\hat{d}^{m} f_{n}(\xi)(x)=\hat{d}^{m} f_{n}(\xi)\left(x^{\prime}\right)$ for each $m$ and $n$. Now $E / \operatorname{ker} p_{L}$ endowed with the quotient topology $s$ is isomorphic to the Banach space $c_{0}(L)$ and putting $\tilde{P}_{n}^{m}\left(x+\operatorname{ker} p_{L}\right):=(1 / m !) \hat{d}^{m} f_{n}(\xi)(x)$ for all $x \in c_{0}(I), m$ and $n$, we have that $\tilde{P}_{n}^{m}$ is an $m$-homogeneous continuous polynomial. Using that $c_{0}(L)$ is a Banach space it is easy to conclude that $\left(f_{n}: n \in \mathbf{N}\right)$ is locally bounded.

4.5. PROPOSITION. Let $U$ be an open subset of a separable space $E$. If $\mathcal{X}$ is a pointwisely bounded subset of $H(U)$ such that every countable subset of $\chi$ is locally bounded, then $\mathcal{X}$ is also locally bounded.

PROOF. Fix $x_{0} \in U$. We prove that $\mathcal{X}$ is bounded on a certain neighbourhood of $x_{0}$ contained in $U$. By assumption $P:=\sup \left(\left|f\left(x_{0}\right)\right|: f \in \mathcal{X}\right)$ is finite. For each $n \in \mathbf{N}$ we set $B_{n}:=\{x \in U:|f(x)| \leq n+P$ for each $f \in X\}$. Clearly $U \backslash B_{n}$ is an open subset of $U$. If $U \backslash B_{n}$ is void for some $n$, then $\chi$ is already bounded on $U$. So we assume $U \backslash B_{n} \neq \varnothing$ for each $n \in \mathbf{N}$. Since $E$ is separable, for each $n$ there is a countable subset $\left(x_{n}^{k}: k \in \mathbf{N}\right)$ of $U \backslash B_{n}$ such that $U \backslash B_{n}$ is contained in the closure of $\left(x_{n}^{k}: k \in \mathbf{N}\right)$ in $E$. For each $k, n \in N$, we select $f_{n}^{k} \in \mathcal{X}$ with $\left|f_{n}^{k}\left(x_{n}^{k}\right)\right|>n+P$. By assumption the countable subset $\left(f_{n}^{k}: k, n \in \mathbf{N}\right)$ of $\chi$ is locally bounded, hence there are an open neighbourhood $V$ of $x_{0}$ included in $U$ and $M>0$ with $\left|f_{n}^{k}(x)\right| \leq M$ for every $k, n \in \mathbf{N}$ and $x \in V$. Now we see that if $m \in \mathbf{N}$ satisfies that $m+P>M$, then $V \subset B_{m}$, hence $B_{m}$ is a neighbourhood of $x_{0}$ and the proof is complete. To show that $V \subset B_{m}$, observe that $\left|f_{m}^{k}\left(x_{m}^{k}\right)\right|>m+P>m$ for every $k \in \mathbf{N}$, hence $x_{m}^{k} \notin V$ for every $k \in \mathbf{N}$. Therefore $\left(x_{m}^{k}: k \in \mathbf{N}\right)$ is included in the closed set $E \backslash V$. Taking closures we have that $U \backslash B_{m}$ is contained in $E \backslash V$, and this implies that $V$ is included in $B_{m}$. 
Various further generalisations may be obtained by using variants of barrelledness etc. (e.g. $\aleph_{0}$-barrelled spaces) and by replacing holomorphic mappings by polynomial mappings in certain cases. The proofs are similar to the proofs given here and we leave the details to the interested reader.

\section{REFERENCES}

1. J. M. Ansemil, Topologies associated with the compact open topology on $H(U)$, Proc. Roy. Irish Acad. Sect. A 82 (1982), 121-128.

2. J. Aragona, On the holomorphical classification of spaces of holomorphic germs, Nagoya Math. J. 84 (1981), 85-118.

3. J. Arias de Reyna, Normed barely Baire spaces, Israel J. Math. 42 (1982), 33-36.

4. J. A. Barroso, M. C. Matos and L. Nachbin, On bounded sets of holomorphic mappings, Proc. Infinite Dimensional Holomorphy (Eds., T. L. Hayden and T. J. Suffridge), Lecture Notes in Math., vol. 364, 1974, pp. 123-134.

5. __ On holomorphy versus linearity in classifying locally convex spaces, Infinite Dimensional Holomorphy and Applications (Ed., M. C. Matos), North-Holland Math. Studies, vol. 12, North-Holland, 1977, pp. 31-74.

6. J. Bochnak and J. Siciak, Analytic functions in topological vector spaces, Studia Math. 39 (1971), 77-112.

7. J. Bonet, The countable neighbourhood property and tensor products, Proc. Edinburgh Math. Soc. (1985), 207-215.

8. S. B. Chae, Holomorphy and calculus in normed spaces, Marcel Dekker, New York, 1985.

9. J. F. Colombeau and J. Mujica, Existence of holomorphic mappings with prescribed asymptotic expansions at a given set of points in infinite dimensions, Nonlinear Anal., Theory, Methods and Applications 5 (1981), 149-156.

10. M. de Wilde et J. Schmets, Caracterisation des espaces $C(X)$ ultrabornologiques, Bull. Soc. Roy. Sci. Liege 40 (1971), 119-121.

11. S. Dineen, Holomorphic functions on locally convex topological vector spaces, I: Locally convex topologies on $H(U)$, Ann. Inst. Fourier (Grenoble) 23 (1973), $19-54$.

12. __ Holomorphic functions on locally convex topological vector spaces, II: Pseudo-convex domains, Ann. Inst. Fourier (Grenoble) 23 (1973), 155-185.

13. __ Surjective limits of locally convex spaces and their application to infinite dimensional holomorphy, Bull. Soc. Math. France 103 (1975), 441-509.

14. _ Holomorphic functions on strong duals of Fréchet-Montel spaces, Infinite Dimensional Holomorphy and Applications (Ed., M. C. Matos), North-Holland Math. Studies, vol. 12, North-Holland, 1977, pp. 147-166.

15. __ Complex analysis in locally convex spaces, North-Holland Math. Studies, vol. 57, NorthHolland, 1981.

16. (1986), 143-146.

17. R. Engelking, General topology, PNW, Warsaw, 1977.

18. K. Floret, Some aspects of the theory of locally convex inductive limits, Functional Analysis, Surveys and Recent Results II (Eds., K. D. Bierstedt and B. Fuchsteiner), North-Holland Math. Studies, vol. 38, North-Holland, 1980, pp. 205-237.

19. P. Galindo, D. Garcı́a and M. Maestre, Holomorphically ultrabornological spaces and holomorphic inductive limits, J. Math. Anal. Appl. 124 (1987), 15-26.

20. A. Grothendieck, Sur les espaces $(F)$ et $(D F)$, Summa Brasil. Mat. 3 (1954), 57-123.

21. H. Jarchow, Locally convex spaces, Teubner, Stuttgart, 1981.

22. G. Köthe, Topological vector spaces, I, II, Springer-Verlag, 1969 and 1979.

23. M. Maestre, Products of holomorphically relevant spaces, Portugal. Math. (to appear).

24. M. C. Matos, Holomorphic mappings and domains of holomorphy, Monografiás do Centro Brasil. de Pesquisas Fisicas, 27, Cent. Brasil. Pesquisas Fisicas, Rio de Janeiro, 1970.

25. __ Holomorphically bornological spaces and infinite-dimensional versions of Hartogs' theorem, J. London Math. Soc. (2) 17 (1978), 363-368. 
26. L. A. de Moraes, Holomorphic functions on strict inductive limits, Resultate Math. 4 (1981), 201-212.

27. _ Holomorphic functions on holomorphic inductive limits and on the strong duals of strict inductive limits, Functional Analysis, Holomorphy and Approximation Theory, II, (Ed., G. I. Zapata), North-Holland, 1984, pp. 297-310.

28. L. Nachbin, Topological vector spaces of continuous functions, Proc. Nat. Acad. Sci. U.S.A. 40 (1954), 471-474.

29. _ , Topology on spaces of holomorphic mappings, Ergeb. Math. Grenzgeb., 47, SpringerVerlag, 1969.

30. __ A glimpse at infinite dimensional holomorphy, Proceedings on Infinite Dimensional Holomorphy (Eds., T. L. Hayden and T. S. Suffridge), Lecture Notes in Math., vol. 364, SpringerVerlag, 1974, pp. 69-79.

31. _ Some holomorphically significant properties of locally convex spaces, Functional Analysis (Ed., D. Figueiredo), Marcel Dekker, 1976, pp. 251-277.

32. P. Noverraz, Pseudo-convexité, convexité polinomial et domains d'holomorphie en dimension infinie, North-Holland Math. Studies, vol. 3, North-Holland, 1973.

33. P. Perez Carreras and J. Bonet, Barrelled locally convex spaces, North-Holland Math. Studies, vol. 131, North-Holland, 1987.

34. D. P. Pombo, Jr., On polynomial classification of locally convex spaces, Studia Math. 78 (1984), 39-57.

35. T. Shirota, On locally convex vector spaces of continuous functions, Proc. Japan Acad. 30 (1954), 294-298.

36. R. Soraggi, Holomorphic germs on certain locally convex spaces, Ann. Mat. Pura Appl. (4) 144 (1986), 1-22.

37. M. Valdivia, On nonbornological barrelled spaces, Ann. Inst. Fourier (Grenoble) 22 (1972), 27-30.

38. _ , Topics in locally convex spaces, North-Holland Math. Studies, vol. 67, North-Holland, 1982.

39. __ Products of Baire topological vector spaces, Fund. Math. 125 (1985), 71-80.

40. S. Warner, The topology of compact convergence on continuous function spaces, Duke Math. J. 25 (1958), 265-282.

Departamento de matematica Aplicada, e.T.S. Arquitectura, Universidad Politécnica de Valencia, C. De Vera, Valencia 46022, Spain (Current address of José Bonet)

Departamento de Análisis Matemático, Facultad de Matemáticas, C/ Dr. Moliner 50, Burjasot 46100 Valencia, Spain (Current address of Pablo Galindo, Domingo García, and Manuel Maestre) 